\title{
Über gepaarte Cyclamine I: \\ 2-(2-Chinolyl)-cyclamine; Valenzformel des Chinolins \\ von
}

\author{
Alexander P. Smirnoff.
}

(20. VIII. 21.)

Zwillingssysteme von stickstoffhaltigen Ringen nehmen unter den bis jetzt in ihrem Bau erkannten Alkalö̈den einen wichtigen Platz ein. Abgesehen von Derivaten der eigenartigen Ringsysteme der Tropan-, Granatan- und Puringruppen weisen viele wichtige Pflanzenbasen Kombinationen von einfach bezw. doppelt verketteten Cyclaminkernen auf; es sei hier nur an die Tabak- und Chinabasen und an das Cuskhygrin erinnert.

Es ist wohl denkbar, dass gegenseitige Substitution von Cyclaminresten Verstärkung gewisser physiologischer Wirkungen bedingen kann, welche den Komponenten in nur untergeordnetem Masse eigen sind; es ist aber auch Auslösung von neuen Effekten möglich.

Es erschien mir daher nicht ohne Wert, Grundsysteme von Cyclaminzwillingen synthetisch darzustellen, um einerseits zu neuen Vergleichstypen für die Erforschung der Abbauprodukte von Alkalö̈den unbekannter Konstitution zu gelangen, andererseits aber diese Substanzen der physiologischen Untersuchung zugänglich zu machen, denn man findet in der Literatur leider recht wenig Körper dieser Art beschrieben.

Die vorliegende Mitteilung betrifft vier einfache Zwillinge aus Chinolin als Hauptsystem mit Pyridin, Pyrrol, Imidazol und Chinolin als Nebensystem, und zwar ausschliesslich mit gegenseitiger Bindung in $(2 \alpha)$-Stellung.

Bei der Darstellung von 2-Pyrryl- resp. 2-Imidazolyl-chinolin-2 konnte direkte Substitution durch Einwirkung der betreffenden sekundären Basen resp. ihrer Kalium- bezw. Silbersalze auf 2-Chlorchinolin, und nachfolgende Umlagerung der primär gebildeten $\mathrm{N}$-Derivate (I) durchgeführt werden, während das genannte Pyrrolderivat sowohl, als namentlich das 2-(2-Pyridyl)-chinolin und das 2-(2-Chinolyl)-chinolin durch Kondensation von o-Aminobenzaldehyd mit den entsprechenden Cyclamin-2-methylketonen unter Synthese eines Chinolinringes erhalten wurden. Die nachstehenden Formeln veranschaulichen z. B. die beiden Synthesen von 2-(2-Pyrryl)-chinolin (II). 
1)

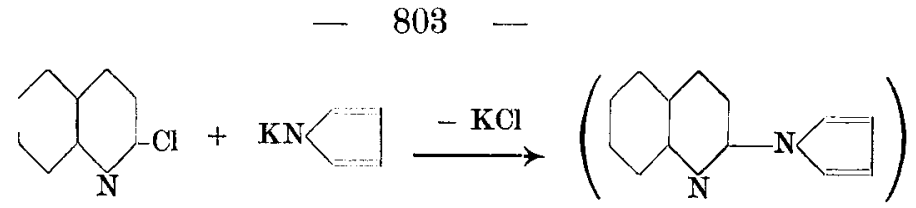

I.

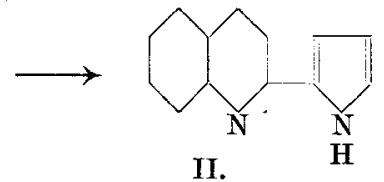

2)

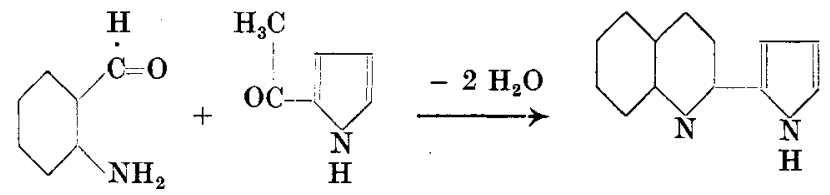

Alle vier Zwillingssysteme stellen sehr beständige, gut krystallisierende Körper dar, welche im allgemeinen die chemischen Eigenschaften der Komponenten zeigen (vgl. immerhin den experimentellen Teil). Die neuen Basen liefern mit Säuren mehr oder minder leicht hydrolysierbare Salze, welche in der Regel sehr leicht löslich sind. In Übereinstimmung mit der Basizität der Komponenten bilden sie folgende (inbezug auf die Basizität ansteigende) Reihe:

$$
\begin{aligned}
& \text { 2-(2-Pyrryl)-chinolin; } \\
& \text { 2-(2-Chinolyl)-chinolin; } \\
& \text { 2-(2-Pyridyl)-chinolin; } \\
& \text { 2-(2-Imidazolyl)-chinolin. }
\end{aligned}
$$

$\mathrm{Zu}$ Identifizierungszwecken wurden die schwer löslichen bezw. unlöslichen Pikrate, Tetrachloro-auriate und Hexachloroplateate dargestellt.

Das Imidazolderivat vermag gleich dem Imidazol selbst ein Silbersalz zu bilden, woraus seine Konstitution als 2-(2-Imidazolyl)-chinolin hervorgeht; die Konstitution von drei anderen Basen ergibt sich ohne weiteres aus ihrer Synthese aus o-Aminobenzaldehyd und Cyclamin2-methylketonen.

Bemerkenswert ist eine feine Differenzierung des Valenzzustandes des Stickstoffatoms im Chinolin- resp. im Pyridinring, welche an Hand des hier beschriebenen Materials deutlich zutage tritt. Während nämlich das 2,2'-Dipyridyl (IV) prächtig blutrote komplexe Ferrosalze der Formel $\left[\mathrm{Fe}\left(\right.\right.$ Dipy $\left._{3}\right] \mathrm{X}_{2}$ bildet $\left.{ }^{1}\right)$, geht diese Eigenschaft dem analogen 2-(2-Pyridyl)-chinolin und dem 2-(2-Chinolyl)-chinolin gänzlich ab; an-

1) F. Blau, M. 19, 650 (1899). 
dererseits ist aber das sog. o-Phenanthrolin (V) zu jener Komplexbildung voll befähigt' ${ }^{1}$. Worin mag nun der Grund dazu liegen?

Berücksichtigt man die Tatsache, dass bei der Oxydation von Chinolin in neutraler oder alkalischer Lösung der Benzolring zerstört wird, während in saurer Lösung die Pyridinseite der Oxydation anheimfällt, so muss man annehmen, dass im Chinolinsystem ein ähnlicher Valenzzustand herrscht, wie im Naphtalin nach Willstätter ${ }^{2}$ ), so zwar, dass im freien Chinolin der echte, ,,aromatische" Pyrjdinring vorhanden ist, die Base aber eigentlich keinen echten Benzolring enthält: dieser trägt eben einen ,,aliphatischen" Charakter.

Nun besitzt aber das Stickstoffatom sowohl im Pyridin- wie im Chinolinring einen noch nicht abgesättigten Valenzbetrag, welcher eben der Träger der Additionsfähigkeit ist. Während aber dieser im - nach Bamberger ${ }^{3}$ ) und v. Pechmann ${ }^{4}$ - völlig symmetrisch gedachten Pyridinkern (III) zu ungehinderter Auswirkung kommt, wird er im Chinolinkern (VI) durch die eine, räumlich sehr nahe Nebenvalenz der konjugierten Doppelbindung der Benzolseite weitgehend abgesättigt, was schon im schwächeren Additionsvermögen und in der geringeren Basizität zum Ausdruck kommt. Dieser Umstand bringt es mit sich, dass weder 2-(2-Pyridyl)-chinolin noch 2-(2-Chinolyl)-chinolin zur erwähnten Komplexbildung befähigt sind, wie aus den Formelbildern VII und VIII für diese Basen ersichtlich ist.

Verfolgt man denselben Gedankengang inbezug auf das o-Phenanthrolin (V), so wird man zugeben müssen, dass dieser Körper näher $\operatorname{dem}$ 2,2'-Dipyridyl steht, als dem 2-(2-Chinolyl)-chinolin (VIII), indem seine aliphatisch-ungesättigte Brücke ohne Einfluss auf die Restvalenzen der beiden Stickstoffatome ist. Das Verhalten der Base stimmt mit dieser Auffassung überein; sie bildet gleich dem 2,2'-Dipyridyl komplexe Ferrosalze von blutroter Farbe und von analoger Zusammensetzung:

$$
\left[\mathrm{Fe}(\mathrm{o}-\mathrm{Phn})_{3}\right] \mathrm{X}_{2}
$$

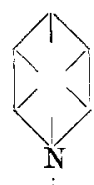

III.

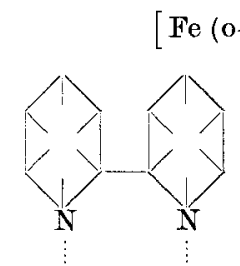

IV.

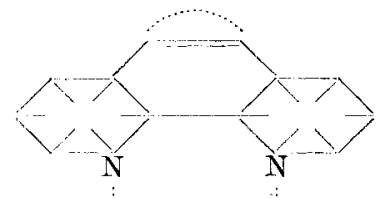

V.

1) Derselbe, 1. c. 668 .

2) R. Willstätter und E. Waser, B. 44, 3430 (1911); vergl. C. Harries, A. 343, 337 (1905).

3) E. Bamberger, B. 24, 1763 (1891).

4) H. v. Pechmann, B. 24, 3152 (1891). 


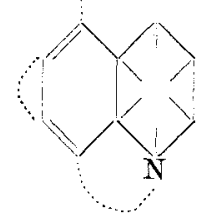

VI.

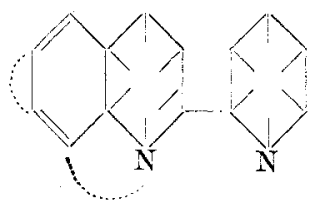

VII.

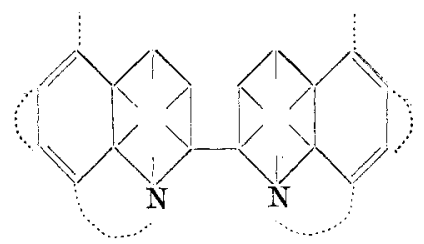

VIII.

Aus denselben Gründen ist zu erwarten, dass die drei möglichen, noch unbekannten Di-isochinoline mit direkter Bindung an den dem Stickstoffatom benachbarten Stellen in ähnlicher Weise Eisenkomplexsalze bilden können, wie das o-Phenanthrolin.

Es bleibt hier noch der wichtige Einwand zu erörtern, dass bei der Hydrierung von Chinolin zuerst die Pyridinseite Wasserstoff anlagert, und somit gerade diese ,aliphatisch" sein müsste. Indessen widerspricht jene Tatsache in keiner Weise der oben entwickelten Vorstellung, wenn man vor allem berücksichtigt, dass die Valenzzustände innerhalb der besprochenen Ringsysteme, für welche unsere Formeln ein grobes Bild zu geben versuchen, nichts Starres sein können, sondern sich in Abhängigkeit von den hinzutretenden Substituenten verändern. Es wird dann die Annahme verständlich sein, dass eine lockere Anlagerung von Wasserstoffatomen an die Nebenvalenzbrücke zwischen dem Stickstoffatom 1 und dem Kohlenstoffatom 8 des Chinolinkernes(VI) genügen kann, um eine Verschiebung des Valenzzustandes im Sinne der Formel IX zu bewirken, worauf die eigentliche Hydrierung an den nun ,aliphatischen" Lücken der Pyridinseite leicht erfolgen kann. In den Fällen, wo sich die Hydrierung in Anwesenheit von Wasserstoffionen, z. B. in salzsaurer Lösung, vollzieht, ist auch diese Annahme überflüssig, weil man es unter diesen Umständen mit neugebildeten komplexen Ionen der Formel X zu tun hat, in welchen die Pyridinseite unbestritten aliphatisch-ungesättigten Charakter hat, wofür ja auch die Ergebnisse der Oxydation von Chinolin in saurer Lösung sprechen, bei welcher die Pyridinhälfte zerstört wird.

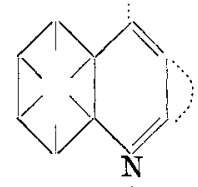

IX. H

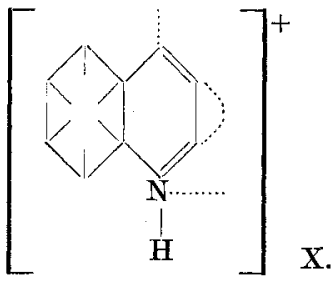

$\mathrm{X}$. 


\section{Experimenteller Teil.}

2-(2-Pyridyl)chinolin.

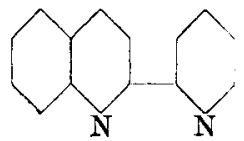

Das als Ausgangsmaterial benötigte 2-Pyridylmethylketon wurde nach Engler und Rosumoff ${ }^{1}$ ) durch trockene Destillation eines Gemisches von picolinsaurem und essigsaurem Calcium dargestellt; es siedete bei $190-192^{\circ}$. Das zweite Material, der o-Aminobenzaldehyd, wurde in allen Fällen nach der Vorschrift von Bamberger und Demuth ${ }^{2}$ ) gewonnen; er schmolz bei $39^{\circ}$.

$4,84 \mathrm{gr}$ o-Aminobenzaldehyd (4 Mol.) wurden in $30 \mathrm{~cm}^{3}$ Alkohol gelöst, mit'der Lösung von 4,84 gr 2-Pyridylmethylketon (4 Mol.) in $10 \mathrm{~cm}^{3}$ Alkohol vermischt und nach Zusatz von $3 \mathrm{~cm}^{3} \mathrm{n}$. Kalilauge eine Stunde lang im Wasserbade am Rückflusskühler erhitzt. Hierauf wurde die Lösung mit Tierkohle entfärbt, filtriert und bis zur Trübung mit heissem Wasser verdünnt. Beim Stehen krystallisierte das 2-(2-Pyridyl)-chinolin in Form von farblosen, anscheinend rhombischen Krystallen aus. Ausbeute $7 \mathrm{gr}$. Die Base ist unlöslich in Wasser, gut löslich in organischen Solventien; Smp. 9!9 .

0,1393 gr Subst. gaben 0,4154 gr $\mathrm{CO}_{2}$ und 0,0602 gr $\mathrm{H}_{2} \mathrm{O}$

0,1228 gr Subst. gaben $14,9 \mathrm{~cm}^{3} \mathrm{~N}_{2}\left(17^{0}, 732 \mathrm{~mm}\right)$

$$
\begin{array}{lllll}
\mathrm{C}_{\mathbf{1 4}} \mathrm{H}_{10} \mathrm{~N}_{2} & \text { Ber. C 81,51 } & \text { H 4,89 } & \text { N } 13,60 \% \\
& \text { Gef. " 81,33 } & \text { "4,8; } & \text { " } 13,76 \%
\end{array}
$$

Eine alkoholische Lösung der Base gilot mit Ferrosulfat keine Färbung.

$$
\text { Salze des 2-(2-Pyridyl)-chinolins. }
$$

Zur Darstellung des Platin- resp. Goldwalzes wurde hier wie weiterhin eine salzsaure Lösung der Base mit einer wässerigen Lösung von Platinchlorwasserstoffsäure resp. von Kaliumtetrachloro-auriat versetzt, wobei sogleich das entsprechende Salz auskrystallisierte. Die Pikrate wurden durch Vermischen alkoholischer Lösungen der Komponenten und durch Umkrystallisieren des erhaltenen Niederschlags dargestellt.

Das Hexachloroplateat bildet sehr schwer lösliche, hellorangegelbe Nädelchen vom Smp. $197^{\circ}$ unter Zersetzung.

1) C. Engler und P. Rosumoff, B. 24, 2527 (I891).

2) E. Bamberger und $O$. Demuth, B. 34, 1329 (1901). 
Das Tetrachloro-auriat stellt hellgelbe Nädelchen von der Feinheit des Asbestes vor, welche beinahe unlöslich in siedendem Wasser sind und bei $211^{\circ}$ unter Zersetzung schmelzen.

Das Pikrat krystallisiert aus wässerig-alkoholischer Lösung in grünlichgelben Täfelchen vom Smp. $183^{\circ}$.

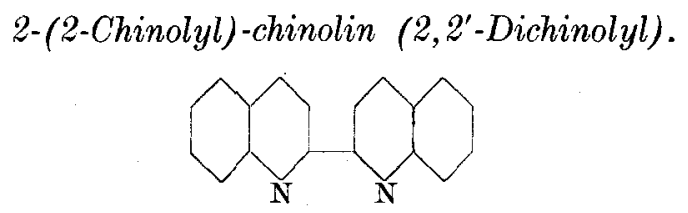

Diese Base konnte durch Kondensation sowohl von 1 Mol. o-Aminobenzaldehyd mit $1 \mathrm{Mol}$. 2-Chinolyl-methylketon als auch von $2 \mathrm{Mol}$. o-Aminobenzaldehyd mit $1 \mathrm{Mol}$. Diacetyl erhalten werden.

a) Kondensation von o-Aminobenzaldehyd mit 2-Chinolylmethylketon.

Das Keton wurde nach Kaufmann und Burkhardt dargestellt1); Smp. 51-52 ${ }^{\circ}$ 2,42 gr o-Aminobenzaldehyd (2 Mol.) und 3,46 gr 2-Chinolylmethylketon wurden zusammen in $50 \mathrm{~cm}^{3}$ Alkohol gelöst und nach Zusatz von $5 \mathrm{~cm}^{3} \mathrm{n}$. Kalilauge im Wasserbade am Rückflusskühler eine Stunde lang erhitzt. Die hierauf mit Tierkohle entfärbte und filtrierte Lösung wurde bis zur Trübung mit heissem Wasser versetzt. Die Base krystallisierte bald in Form von kleinen Blättchen aus. Nach einmaligem Umkrystallisieren aus heissem Alkohol stellte das 2,2'-Dichinolyl dünne, glimmerglänzende Blättchen von unregelmässigem Umriss vor. Smp. $196^{\circ}$. Ausbeute 4 gr. Die Base ist unlöslich in Wasser, und etwas weniger löslich in den organischen Solventien als das 2-(2-Pyridyl)-chinolin.

0,1436 gr Subst. gaben $0,4433 \mathrm{gr} \mathrm{CO}_{2}$ und $0,0598 \mathrm{gr}_{2} \mathrm{O}$

0,1328 gr Subst. gaben $13 \mathrm{~cm}^{3} \mathrm{~N}_{2}\left(18^{0}, 728 \mathrm{~mm}\right)$

$\begin{array}{rlrrr}\mathrm{C}_{18} \mathrm{H}_{12} \mathrm{~N}_{2} & \text { Ber. C 84,34 } & \mathrm{H} 4,72 & \mathrm{~N} 10,94 \% \\ & \text { Gef. }, 84,19 & \text { " } 4,68 & \text {, } 11,07 \%\end{array}$

Die Verbindung gibt keine Färbung mit Ferrosulfat.

b) Kondensation von o-Aminobenzaldehyd mit Diacetyl.

$4,84 \mathrm{gr}$ o-Aminobenzaldehyd (4 Mol.) wurden in $50 \mathrm{~cm}^{3}$ Alkohol gelöst, mit 2 gr Diacetyl (etwas mehr als 2 Mol.) und darauf mit $5 \mathrm{~cm}^{3}$ $\mathrm{n}$. Kalilauge versetzt und gut verschlossen an einem mässig warmen

1) A. Kaufmann und H. Burkhardt, B. 46, 2931 (1913). 
Ort 6 Stunden lang der Ruhe überlassen. Schliesslich wurde noch eine Stunde lang am Rückflusskühler im Wasserbade erhitzt und dann weiter wie unter a) verfahren. Ausbeute an reiner Base 3 gr. Smp. $196^{\circ}$; die Mischprobe mit der Substanz ler ersten Darstellung zeigte keine Depression.

\section{Salze des 2,2'-Dichinolyls.}

Das unlösliche Hexachloroplateat billet kleine, hell orangegelbe Blättchen. Beim Erhitzen verfärbt sich das Salz allmählich (von ca. $200^{\circ}$ an) braun, schmilzt aber bei $300^{\circ}$ noch nicht.

Das schwer lösliche Tetrachloro-auriat fällt als voluminöser Niederschlag aus, welcher sich bald - besonders rasch beim Erwärmen in feine gelbe Nädelchen verwandelt. Bein Trocknen zersetzt sich das Salz unter Dunkelfärbung.

Das Pikrat stellt intensiv gelbe feine Nädelchen rom Smp. $210^{\circ}$ vor; es ist ziemlich leicht löslich in heissem Wasser und in heissem Alkohol.

\section{2-(2-Pyrryl)-chinolin:}

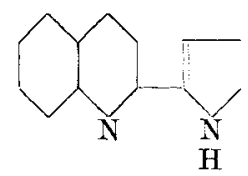

Auch diese Base wurde auf zweierlei Weisen synthetisch dargestellt: lurch Kondensation von 2-Chlorchinolin mit Pyrrolkalium und nachfolgende Umlagerung des primär entstandenen 2-(1-Pyrryl)-chinolins einerseits, und durch Kondensation von o-Aminobenzaldehyd mit 2-Pyrryl-methylketon andererseits. Nach beiden Methoden wurde ein und dieselbe Base erhalten; für die Konstitution der Verbindung als 2-(2-Pyrryl)-chinolin ist die zweite Synthese beweisend.

a) Kondensation von 2-Chlorchinolin mit Pyrrolkalium.

2-Chlorchinolin wurde aus Carbostyril nach Friedländer und Ostermaier gewonnen ${ }^{1}$ ); Smp. $37^{\circ}$.

In einem offenen Einschlussrohr wurden 1,2 gr Kalium mit 2,5 gr Pyrrol, gelöst in $10 \mathrm{~cm}^{3}$ Xylol, bei $130^{\circ}$ unter Ausschluss der Feuchtigkeit zur Reaktion gebracht. Als keine Wasserstoffentwicklung mehr

1) P. Friedländer und H. Ostermaier, B. 15, 333 (1882). 
stattfand, liess man die Masse erkalten, goss das pyrrolhaltige Xylol ab, wusch die Krusten von Pyrrolkalium rasch mit trockenem Benzol aus und entfernte die Reste von Benzol durch Absaugen bei schwach angewärmtem Rohr. Hierauf wurden $5 \mathrm{gr}$ (etwas mehr als berechnet) 2-Chlorchinolin hinzugefügt; das Rohr wurde nun zugeschmolzen und 6 Stunden lang auf $230^{\circ}$ erhitzt. Nach dem Öffnen bestand der Rohrinhalt aus einer dunklen, voluminösen Masse von krystallinischem Bruch, welche sich leicht pulverisieren liess. Das Pulver wurde wiederholt mit schwach angesäuertem Wasser behandelt, um Kaliumchlorid und unverbrauchtes 2-Chlorchinolin zu entfernen. Schliesslich wurde die Masse in Alkohol gelöst und mit Tierkohle gekocht. Die filtrierte, gelbliche Lösung wurde bis zur Trübung mit heissem Wasser versetzt. Bald begann die Abscheidung von schwach gelblichen Blättchen, welche nach 12 Stunden abgesaugt, getrocknet und aus heissem Petroläther umkrystallisiert wurden. Die so gewonnene Base stellte kleine, sechseckige, fettglänzende Blättchen vor, welche bei $129^{\circ}$ schmelzen. Ausbeute $4 \mathrm{gr}$.

b) Kondensation von o-Aminobenzaldehyd mit 2-Pyrryl-methylketon.

Das Keton wurde nach der von Tschelinzeff und Terentjeff ${ }^{1}$ ) modifizierten Methode von $O d d 0^{2}$ ) gewonnen. 2,42 gr o-Aminobenzaldehyd (2 Mol.) wurden in $20 \mathrm{~cm}^{3}$ Alkohol gelöst, mit der Lösung von 2,18 gr 2-Pyrryl-methylketon (2 Mol.) in $15 \mathrm{~cm}^{3}$ Alkohol versetzt und nach Zusatz von $3 \mathrm{~cm}^{3} \mathrm{n}$. Kalilauge eine Stunde lang im Wasserbade am Rückflusskühler erhitzt. Man entfärbte dann die Lösung mit Tierkohle, filtrierte, verdünnte mit heissem Wasser bis zur Trübung und verfuhr weiter wie unter a). Die schliesslich erhaltene Base glich vollkommen jener aus der ersten Darstellung; allerdings war sie nicht völlig von einem gelblichen Stich zu befreien. Trotzdem stimmte der Schmelzpunkt $129^{\circ}$ überein; die Mischprobe zeigte keine Depression. Ausbeute 3,5 gx.

0,1384 gr Subst. gaben $0,4075 \mathrm{gr}^{\mathrm{CO}_{2}}$ und 0,0635 gr $\mathrm{H}_{2} \mathrm{O}$

0,1207 gr Subst. gaben $15,7 \mathrm{~cm}^{3} \mathrm{~N}_{2}\left(19^{0}, 732 \mathrm{~mm}\right)$

$$
\begin{array}{lllll}
\mathrm{C}_{13} \mathrm{H}_{10} \mathrm{~N}_{2} & \begin{array}{l}
\text { Ber. C } 80,37 \\
\text { Gef. }
\end{array} & \mathrm{H} & 5,19 & \mathrm{~N} 14,44 \% \\
& \text { Ge,30 } & \text {, } 5,13 & , 14,65 \%
\end{array}
$$

Die Base ist unlöslich in Wasser, gut löslich in organischen Solventien. Sie besitzt einen schwachen, angenehmen Geruch, ähnlich

1) W. Tschelinzeff und A. Terentjeff, B. 47, 2647 (1914).

2) B. Oddo, G. 39, I, 649 (1909). 
dem des Nerolins. Obwohl sie zweifellos ein Pyrrolderivat ist, gibt sie keine Fichtenspanreaktion von roter Farbe, sondern färbt das Holz intensiv gelb an; dieselbe Farbe besitzen ihre Lösungen in konzentrierten Säuren. Beim Verdünnen solcher gelber Lösungen mit Wasser fällt die schwache Base fast unverändert wieder aus; ein Teil scheint dabei immerhin zu verharzen, denn die Fällung ist mehr oder weniger gelb gefärbt, sie kann jedoch ohne wesentlichen Verlust wie angegeben gereinigt werden.

$$
\text { Salze des 2-(2-Pyrryl)-chinolins. }
$$

Von diesen konnte nur das Hexachloroplateat krystallinisch gewonnen werden. Es stellt schwer lösliche, gelbbraune Prismen vor, welche sich bei $210^{\circ}$ unter Șchwarzfärbung zersetzen.

Das gelbe Goldsalz zersetzt sich in kurzer Zeit nach der Bildung unter Goldausscheidung. Pikrinsäure bildet mit der Base in wässeriger Lösung kein Salz.

\section{2-(2-Imidazolyl)-chinolin:}

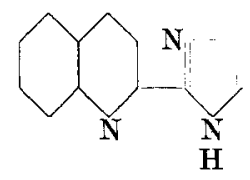

Das aus 2,72 gr Imidazol (4 Mol.) bereitete und im Dunkeln getrocknete Silbersalz wurde mit 6,5 gr 2-Chlorchinolin (4 Mol.) 2 Stunden lang im Ölbade auf $180^{\circ}$ erhitzt. Die abgekühlte Schmelze wurde in verdünnter Salpetersäure aufgenommen, wobei graues Silberchlorid ungelöst blieb. Die von diesem abfiltrierte salpetersaure Lösung war silberfrei, was für einen vollständigen Umsitz spricht. Man entfärbte die Lösung, soweit es ging, mit Tierkohle, filtrierte und machte das Filtrat mit Natronlauge alkalisch, wobei ein weisser Niederschlag entstand. Dieser wurde abgesaugt, mit Wasser gewaschen und in heissem Alkohol gelöst; die filtrierte Lösung wurde mit heissem Wasser bis zur Trübung versetzt. Beim Stehen krystallisierte die Base in Form von schönen, glänzenden Prismen aus, die jedoch schon an der Luft unter Wasserabgabe rasch mattweiss wurden. Ausbente 6,4 gr.

Das 2-(2-Imidazolyl)-chinolin schmilzt bei $119^{\circ}$; es ist schwer löslich in Wasser, leicht löslich in organischen Solventien. Es ist unter den hier beschriebenen Basen die stärkste. Es ist ein 2-Imidazolylderivat, denn es gibt mit ammoniakalischer Silbernitratlösung ein 
weisses Silbersalz, das jedoch weit löslicher als Imidazolsilber ist; die Base muss somit durch Umlagerung des primär gebildeten 2-(1-Imidazolyl)-chinolins entstanden sein.

Zur Analyse wurde die Substanz über Schwefelsäure getrocknet.

0,1244 gr Subst. gaben $0,3360 \mathrm{gr}^{\mathrm{CO}_{2}}$ und 0,0511 gr $\mathrm{H}_{2} \mathrm{O}$

$0,1096 \mathrm{gr}$ Subst. gaben $21,1 \mathrm{~cm}^{3} \mathrm{~N}_{2}\left(16^{0}, 726 \mathrm{~mm}\right)$

$\mathrm{C}_{12} \mathrm{H}_{9} \mathrm{~N}_{3}$ Ber. C 73,81 H 4,65 N 21,54\%

Gef. „73,66 , 4,59 „21,72\%

Salze des 2-(2-Imidazolyl)-chinolins.

Das Hexachloroplateat bildet schöne orangefarbene Prismen, welche schwer lisslich sind. Smp. über $330^{\circ}$ unter Zersetzung.

Das Tetrachloro-auriat stellt goldgelbe Blättchen vor; Smp. $205^{0}$ unter Zersetzung.

Das Pikrat krystallisiert in langen, dünnen, seidenglänzenden Nadeln vom Smp. $201^{\circ}$.

Zürich, Elektrochemisches Laboratorium der Eidg. Techn. Hochschule. 27. Juli 1921.

\title{
Polysaccharide $\mathrm{XI}^{1}{ }^{1}$.
}

Die Verbindungen der Anhydrozucker mit Alkalilaugen. Eine Methode zur Bestimmung der Grundkörper polymerer Anhydrozucker

\author{
von \\ P. Karrer. \\ (24. VIII. 21.)
}

Kürzlich machte ich die Beobachtung ${ }^{2}$ ), dass sich die in Wasser sehr schwer löslichen Verbindungen $\beta$-Hexamylose und $\alpha$-Oktamylose in verdünnter Natronlauge spielend auflösen. Die genauere Untersuchung dieser Erscheinung hat zu einem praktisch wichtigen Resultat geführt.

1) X. Mitteilung Helv. 4, 796 (1921).

2) Helv. 4, 678 (1921). 